\title{
Tamoxifen: From Anti-cancer to Antifungal Drug
}

\author{
Ali Abdul Hussein S. AL-Janabi ${ }^{*}$, Huda Ali S. Al-Mosawe', Karrar Al-Mosawi² \\ ${ }^{1}$ Department of Microbiology, College of Medicine, University of Karbala, Karbala, Iraq \\ ${ }^{2} \mathrm{Al}-\mathrm{Ammam} \mathrm{Al}-\mathrm{Hussein}$ Medical City Hospital, Karbala, Iraq
}

Corresponding Author: Ali Abdul Hussein S. AL-Janabi, PhD, Professor, Department of Microbiology, College of Medicine, University of Karbala, Karbala, Iraq. Tel: +964-7811411260, Email: aljanabi_bio@yahoo.com

Received May 5, 2019; Accepted August 28, 2019; Online Published September 16, 2019

\begin{abstract}
Tamoxifen (TAM) is an important drug for treatment of breast cancer. It is most effective against estrogen receptor-positive and negative breast cancer. Protective adjuvant is another applied of TAM for women at risk of development of breast cancer. Anti-cancer activity of TAM can take various pathways of action. Antagonistic with estrogen receptor and oxidation reaction are the most proposed mechanism of action of TAM in cancer cells. Recently, many studies focused on the potential antimicrobial action of TAM. Fungi are demonstrated to affect by TAM through various mechanism of action. Yeasts, especially Candida albicans, are the most common type of fungi used to test the antifungal action of TAM. Inhibitory action on some components of the calcium-calcineurin pathway in fungal cells is most acceptable mechanism of TAM action. TAM can also play a synergistic role to increase the antifungal activity of other standard agents. This review will discuss the most recent information about antifungal action of TAM.

Keywords: Antifungal Agents, Breast Cancer, Estrogen Receptor Alpha, Tamoxifen

Citation: AL-Janabi AAHS, Al-Mosawe HAS, Al-Mosawi K. Tamoxifen: from anti-cancer to antifungal drug. Int J Med Rev. 2019;6(3):88-91. doi:10.29252/ijmr-060304.
\end{abstract}

\section{Introduction}

Tamoxifen $\left(\mathrm{C}_{26} \mathrm{H}_{29} \mathrm{NO}\right.$, TAM), which has $371.524 \mathrm{~g} / \mathrm{mol}$ molecular weight, is a drug member of a group called selective estrogen receptor modulators (SERMs). ${ }^{1}$ It is commonly takes a fine white, odorless crystalline powder which is soluble in ethanol, methanol, or acetone and slightly soluble in water. Other physical characters of TAM include its hygroscopic nature at a high moisture rate and sensitive to UV light. ${ }^{1}$

The main actions of TAM in the human body involve antiestrogenic effects in the mammary tissue, and as an estrogenic effect (stimulating agent) in cholesterol metabolism, bone density, and cell proliferation in the endometrium. ${ }^{1,2}$ It may also act as an antioxidant agent through protect the stability of the liposome membrane of mammalian cell by reduce the fluidity of this membrane. ${ }^{3}$ The concentration of TAM can effect on fluidity rate such that low concentrations reduce membrane fluidity, while it increase at high TAM concentration. ${ }^{4}$ Antimicrobial action of TAM is another new function can add to the list of known actions in organisms.

\section{Tamoxifen as a Treatment of Breast Cancer}

Breast cancer is one of hormone-dependent cancer in the female which is usually stimulated by estrogen hormone., Thus, TAM is successfully used as an anti-estrogenic agent for treatment of breast cancer and also as an adjuvant to protect women at risk of development of breast cancer. ${ }^{7,8}$ Curative results of TAM against breast cancer found more appropriate to use than old surgical process such as oophorectomy, hypophysectomy or adrenalectomy. ${ }^{7}$ The USA-FDA approved usage of TAM in 1977 for the treatment of postmenopausal women suffering from advanced breast cancer and also as postsurgery adjuvant treatment to eradicate micrometastasis from primary breast cancer. ${ }^{9}$ Accumulate of TAM in tumor mass has the ability to kill both types of positive and negative estrogen receptor- $\alpha$ (ER $\alpha$ ) breast cancer. ${ }^{10}$ It cured 5\%-10\% of females with ER-negative breast cancers with depending on many factors such as variation in the expression of estrogen-related receptor alpha, ER subtype beta, tumor microenvironment, and epigenetics. ${ }^{11}$ Furthermore, TAM proved to reduce recurrence and mortality of the estrogen receptor positive breast cancer by half. ${ }^{12}$ A dose of $20 \mathrm{mg}$ TAM twice daily is more effective to arrest or reversal of $77 \%$ of advanced, recurrent or metastatic breast carcinoma compared with a dosage of $10 \mathrm{mg}(60 \%){ }^{13}$ This dosage (20 $\mathrm{mg}$ ) is also recommended to use for 5 years, when TAM applied as adjuvant chemotherapy for improving treatment of premenopausal patients with only ER-positive tumor and not those with ER-negative tumor. ${ }^{8}$ However, the usual therapeutic period of TAM for treatment of breast cancer may take at least 5 years and can recommend to be given for 10 years if want to reduce more than $20 \%$ of cancer recurrent. ${ }^{5}$

Copyright $\odot 2019$ The Author(s). This is an open-access article distributed under the terms of the Creative Commons Attribution License (http:// creativecommons.org/licenses/by/4.0), which permits unrestricted use, distribution, and reproduction in any medium, provided the original work is properly cited. 
Patients with breast cancer may reveal resistance to treatment with hormonal therapy, including TAM. $^{6}$ The resistance towards TAM is clearly observed after initial 5-10 years of TAM therapy and may give a chance for other cancers to develop during this time as with uterine cancer. ${ }^{14}$ Thus, selecting effective treatment modalities should be optimized according to the stage of disease and the risk. ${ }^{15}$

\section{Mechanism of Action of TAM in Treatment of Breast Cancer}

The therapeutic activity of TAM, mainly depends on inhibition the binding of estrogen with estrogen receptors, which will prevent later effect of estrogen on cellular DNA to form breast cancer. ${ }^{1,7}$ The TAM is also has the ability to direct binding with DNA molecule, but without effect on the DNA confirmation form. ${ }^{16}$ Other activities of TAM against tumor developments are also recorded. Low concentration (in microgram) of TAM may play an inhibitory role in cell growth and a cytotoxic action by effects on protein kinase $\mathrm{C}$ of tumor cells. ${ }^{17}$ It can regulate the action of the inhibitor tumor cell growth factor, such as transforming growth factor B (TGFb) and the stimulate breast cancer cell growth factor, such as insulin-like growth factor 1 (1GF-1). ${ }^{1}$ Tumor growth can reduce by TAM through its inhibitory effects on mitochondrial complex 1 leading to inhibition of oxygen consumption as indicated by an increase of AMP/ATP ration and activation of AMPK signaling pathway. ${ }^{18}$ The antitumor activity of TAM may include its ability to induce an oxidation reaction of the breast cancer cells through increasing of Nrf2 expression, but this activity can also develop TAM a resistance breast cancer. ${ }^{10}$ However, there are many biochemical factors can associate with resistance of breast cancer to TAM treatment such as the modulation in ER signaling and upregulation of growth factors. ${ }^{14}$

\section{Tamoxifen as an Anti-fungal Drug}

Treatment of fungal infection in cancer patients by standard antifungal agents may raise undesirable problems. Several adverse effects and drug interaction are clinically identified after using either of itraconazole, voriconazole or posaconazole for treatment of fungal infection in patients with cancer diseases. ${ }^{19}$ Thus, an additional therapeutic function of known drugs is always required to discover over time, which is called drug repositioning and has the same meaning of drug repurposing or drug reprofiling. ${ }^{20}$ For repurpose of TAM, antifungal action is another potential activity that can add to its therapeutic ability of breast cancer. ${ }^{21}$ Wiseman et al gave the first indicator for antifungal activity of TAM against Saccharomyces cerevisiae. ${ }^{22}$ A few years later, many studies demonstrated that TAM has such activity against other types of fungi. Candida albicans is the most common type of fungi used to test the antifungal action of TAM. Clinical isolates of fluconazole-sensitive and resistance C. albicans from patients with periodontal disease revealed in vitro inhibition by TAM. $^{23}$ In comparison with imidazole, TAM showed fungicidal action against $C$. albicans at low concentration
(15-20 $\mu \mathrm{M})$, especially at stationary phase of yeast growth. ${ }^{24}$ Whereas, the MIC range may become higher $(8-64 \mathrm{~g} / \mathrm{mL})$ for Cryptococcus neoformans and other isolates of Candida spp. ${ }^{25}$ Biofilm formation by $C$. albicans also found to inhibit by 1 $\mathrm{mg} / \mathrm{mL} \mathrm{TAM.}{ }^{26}$ After testing the antifungal effects of TAM on the logarithmic growth of C. albicans, it found that, TAM has fungicidal effects at $2 \times 10^{-5} \mathrm{M}$ and moderate and transient at $1 \times 10^{-5} \mathrm{M}$, while it exhibited very weak action at $5 \times 10^{-6}$ M. ${ }^{27}$ This fungicidal activity of TAM on C. albicans is more sensitive to $\mathrm{pH}$ value as noted when the large inhibitory effect of $10 \mu \mathrm{M}$ TAM (>99\%) was exhibited at higher values of $\mathrm{pH}$ than at the neutral range. ${ }^{28}$

The TAM also exhibited in vivo antifungal action against disseminated candidiasis in a dose of $200 \mathrm{mg} / \mathrm{kg}$ given to murine model. ${ }^{25}$ However, the fungicidal and fungistatic activity of TAM on C. albicans is quite similar to that of

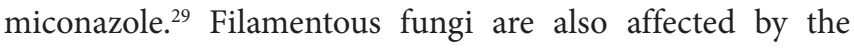
antifungal activity of TAM. Growth of Aspergillus spp., Penicillium spp., Mucor spp., and Rhizopus spp. inhibited in the presence of TAM. ${ }^{30}$

An interaction between TAM and other antifungal agents are observed, when they use together in patients with cancer. According to the results of the physiologically based pharmacokinetic models, triazole agents can increase the pharmacokinetic parameters of TAM when coadministrated with it, especially with voriconazole. ${ }^{31}$ Otherwise, TAM can play a synergistic action to increase the antifungal activity of other agents of azole and terbinafine. ${ }^{32}$ The curative value of amphotericin B (AmB) and fluconazole for Cryptococcosis infection, as with Cryptococcosis meningitis, can also enhance after using with TAM..$^{33,34}$

\section{Mechanism of Action of TAM as Antifungal Agent}

The mechanism of action of TAM as antifungal agents is not clearly demonstrated. Calcium-calcineurin signaling pathway is an important pathway for fungal growth, development and reproduction and it's associated with the virulence of many pathogenic fungi. ${ }^{35}$ The TAM can perform its inhibitory action on some components of the calcium-calcineurin pathway in fungal cells, and making them more sensitive to antifungal drugs such $\mathrm{AmB}$ and fluconazole. ${ }^{36}$ Another proposed mechanism of TAM action on fungal cells, depends on its block effect on calmodulin site..$^{25,37,38}$ Calmodulin, is usually activated the serine-threonine phosphatase calcineurin pathway, which is associated with the virulence of fungi such as Cryptococcus neoformans to cause disease in the human body. ${ }^{39}$ Calmodulin affected by TAM, can be detected in C. albicans through changing in its phenotype characters, including development of cell lysis, decrease new bud formation, disrupted actin polarization and decrease germ tube formation. ${ }^{25}$ However, Krysan and Bucheit hypothesized that, the inhibitory action of TAM in fungal calmodulin is provided by the presence of five evidences based on studies on S. cerevisiae. First, calmodulin mutants cells showed the defect in phenotype morphology, nuclear separation and in actin cytoskeleton after treating with TAM; second, calmodulin 
mutants became hypersensitive to TAM; third, resistance to TAM developed after overexpression of calmodulin in yeast; forth, shifting in the location of calmodulin in TAM-treated yeast cells; fifth, TAM disrupts the binding of proteins to yeast calmodulin according to co-immunoprecipitation studies. ${ }^{38}$

\section{Conclusions}

The potential ability of TAM to inhibit different types of fungi, can introduce a new drug with another choice to treatment fungal infection. Resistance fungi to available antifungal agents, will not be a problem anymore after complete proving the successful antifungal application of TAM.

\section{Authors' Contributions}

AAHSAJ is responsible for design and writing the manuscripts. HASAM is responsible for sample collection and lab work. KAM responsible for diagnosis of patients.

\section{Conflict of Interest Disclosures}

The authors declare they have no conflicts of interest.

\section{References}

1. Tamoxifen. Drug bank website. https://www.drugbank.ca/drugs/ DB00675. Accessed 13 May 2019.

2. National Center for Biotechnology Information (NCBI). Tamoxifen. PubChem website. 2019. https://pubchem.ncbi.nlm.nih.gov/ compound/Tamoxifen.

3. Wiseman $H$, Quinn $P$, Halliwell B. Tamoxifen and related compounds decrease membrane fluidity in liposomes. Mechanism for the antioxidant action of tamoxifen and relevance to its anticancer and cardioprotective actions? FEBS Lett. 1993;330(1):53-56. doi:10.1016/0014-5793(93)80918-k.

4. Severcan F, Kazanci N, Zorlu F. Tamoxifen increases membrane fluidity at high concentrations. Biosci Rep. 2000;20(3):177-184. doi:10.1023/a:1005515502954.

5. Ramani KV, Ramani H, Alurkar S, Ajaikumar BS, Trivedi RG. Breast Cancer, Medical Treatment, Side Effects, and Complementary Therapies. New York: Momentum Press; 2017.

6. Ingvarsson S. Breast cancer: Introduction. Semin Cancer Biol. 2001;11(5):323-326. doi:10.1006/scbi.2001.0381.

7. Osborne CK. Tamoxifen in the treatment of breast cancer. $N$ Engl J Med. 1998;339(22):1609-1618. doi:10.1056/ nejm 199811263392207 .

8. Colleoni M, Gelber S, Goldhirsch A, et al. Tamoxifen after adjuvant chemotherapy for premenopausal women with lymph nodepositive breast cancer: International Breast Cancer Study Group Trial 13-93. J Clin Oncol. 2006;24(9):1332-1341. doi:10.1200/ jco.2005.03.0783.

9. Lazarus P, Sun D. Potential role of UGT pharmacogenetics in cancer treatment and prevention: focus on tamoxifen and aromatase inhibitors. Drug Metab Rev. 2010;42(1):182-194. doi: 10.3109/03602530903208652.

10. Bekele RT, Venkatraman G, Liu RZ, et al. Oxidative stress contributes to the tamoxifen-induced killing of breast cancer cells: implications for tamoxifen therapy and resistance. Sci Rep. 2016;6:21164. doi:10.1038/srep21164.

11. Manna S, Holz MK. Tamoxifen action in ER-negative breast cancer. Sign Transduct Insights. 2016;5:1-7. doi:10.4137/sti.s29901.

12. Aromatase inhibitors versus tamoxifen in early breast cancer: patient-level meta-analysis of the randomised trials. Lancet. 2015;386(10001):1341-1352. doi:10.1016/s01406736(15)61074-1
13. Ward HW. Anti-oestrogen therapy for breast cancer: a trial of tamoxifen at two dose levels. Br Med J. 1973;1(5844):13-14. doi:10.1136/bmj.1.5844.13.

14. Ali S, Rasool M, Chaoudhry H, et al. Molecular mechanisms and mode of tamoxifen resistance in breast cancer. Bioinformation. 2016;12(3):135-139. doi:10.6026/97320630012135.

15. Anjum F, Razvi N, Masood MA. Breast cancer therapy: a mini review. MOJ Drug Des Dev Ther. 2017;1(2):35-38. doi:10.15406/ mojddt.2017.01.00006.

16. Bourassa P, Thomas TJ, Tajmir-Riahi HA. Locating the binding sites of antitumor drug tamoxifen and its metabolites with DNA. J Pharm Biomed Anal. 2014;95:193-199. doi:10.1016/j.jpba.2014.02.028.

17. O'Brian CA, Liskamp RM, Solomon DH, Weinstein IB. Inhibition of protein kinase C by tamoxifen. Cancer Res. 1985;45(6):24622465.

18. Daurio NA, Tuttle SW, Worth AJ, et al. AMPK Activation and Metabolic reprogramming by tamoxifen through estrogen receptorindependent mechanisms suggests new uses for this therapeutic modality in cancer treatment. Cancer Res. 2016;76(11):32953306. doi:10.1158/0008-5472.can-15-2197.

19. Cronin S, Chandrasekar PH. Safety of triazole antifungal drugs in patients with cancer. J Antimicrob Chemother. 2010;65(3):410416. doi:10.1093/jac/dkp464.

20. Doan TL, Pollastri M, Walters MA, Georg G. Chapter 23: The future of drug repositioning: old drugs, new opportunities. Annu Rep Med Chem. 2011;46(17):385-401. doi:10.1016/B978-0-12386009-5.00004-7.

21. Montoya MC, Krysan DJ. Repurposing estrogen receptor antagonists for the treatment of infectious disease. MBio. 2018;9(6). doi:10.1128/mBio.02272-18.

22. Wiseman $H$, Cannon M, Arnstein HR. Observation and significance of growth inhibition of Saccharomyces cerevisiae (A224A) by the anti-oestrogen drug tamoxifen. Biochem Soc Trans. 1989;17(6):1038-1039. doi:10.1042/bst0171038.

23. Muthular M, Balsamo F, Passero P, et al. Effects of tamoxifen on periodontal disease and Candida albicans of patients with breast cancer and other pathologies. Future Microbiol. 2019;14:129137. doi:10.2217/fmb-2018-0272.

24. Beggs $\mathrm{WH}$. Growth phase in relation to the lethal action of tamoxifen on Candida albicans. Res Commun Mol Pathol Pharmacol. 1995;88(1):115-118.

25. Dolan K, Montgomery S, Buchheit B, Didone L, Wellington M, Krysan DJ. Antifungal activity of tamoxifen: in vitro and in vivo activities and mechanistic characterization. Antimicrob Agents Chemother. 2009;53(8):3337-3346. doi:10.1128/aac.01564-08.

26. Wakharde AA, Halbandge SD, Phule DB, Karuppayil SM. Anticancer drugs as antibiofilm agents in Candida albicans: potential targets. Assay Drug Dev Technol. 2018;16(5):232-246. doi:10.1089/adt.2017.826.

27. Beggs WH. Anti-Candida activity of the anti-cancer drug tamoxifen. Res Commun Chem Pathol Pharmacol. 1993;80(1):125-128.

28. Beggs $\mathrm{WH}$. Drug protonation and $\mathrm{pH}$ in relation to the lethal action of tamoxifen on Candida albicans. J Antimicrob Chemother. 1996;37(4):841-842. doi:10.1093/jac/37.4.841.

29. Beggs WH. Comparative activities of miconazole and the anticancer drug tamoxifen against Candida albicans. J Antimicrob Chemother. 1994;34(1):186-187. doi:10.1093/jac/34.1.186.

30. Pasrija R, Kundu D. First report of antifungal activity of tamoxifen against filamentous fungi. Int J Life Sci Res. 2018;6(4):296-298.

31. Chen L, Zhu L, Li M, Li N, Qi F, Wang N. Predicting the effects of different triazole antifungal agents on the pharmacokinetics of tamoxifen. AAPS PharmSciTech. 2019;20(1):24. doi:10.1208/ s12249-018-1219-5.

32. Zhang $X$, Fang $Y$, Jaiseng $W$, et al. Characterization of Tamoxifen as an Antifungal Agent Using the Yeast Schizosaccharomyces Pombe 
Model Organism. Kobe J Med Sci. 2015;61(2):E54-63.

33. Butts A, Koselny K, Chabrier-Rosello Y, et al. Estrogen receptor antagonists are anti-cryptococcal agents that directly bind EF hand proteins and synergize with fluconazole in vivo. MBio. 2014;5(1):e00765-00713. doi:10.1128/mBio.00765-13.

34. Ngan NTT, Mai NTH, Tung NLN, et al. A randomized open label trial of tamoxifen combined with amphotericin B and fluconazole for cryptococcal meningitis. Wellcome Open Res. 2019;4:8. doi:10.12688/wellcomeopenres.15010.1.

35. Rispail N, Soanes DM, Ant C, et al. Comparative genomics of MAP kinase and calcium-calcineurin signalling components in plant and human pathogenic fungi. Fungal Genet Biol. 2009;46(4):287298. doi:10.1016/j.fgb.2009.01.002.
36. Liu S, Hou Y, Liu W, Lu C, Wang W, Sun S. Components of the calcium-calcineurin signaling pathway in fungal cells and their potential as antifungal targets. Eukaryot Cell. 2015;14(4):324-334. doi:10.1128/ec.00271-14.

37. Butts A, Martin JA, DiDone L, Bradley EK, Mutz M, Krysan DJ. Structure-activity relationships for the antifungal activity of selective estrogen receptor antagonists related to tamoxifen. PLoS One. 2015;10(5):e0125927. doi:10.1371/journal.pone.0125927.

38. Krysan D, Buchheit B. Antifungal activity of tamoxifen: Scope and mechanism. 48th Annual ICAAC/46th Annual IDSA NULL Conference abstract; 2008.

39. Odom AR. The triphenylethylenes, a novel class of antifungals. MBio. 2014;5(3):e01126-01114. doi:10.1128/mBio.01126-14. 